\title{
High Order Semi-implicit Multistep Methods for Time-Dependent Partial Differential Equations
}

\author{
Giacomo Albi ${ }^{1} \cdot$ Lorenzo Pareschi $^{2}$
}

Received: 31 December 2019 / Revised: 17 September 2020 / Accepted: 5 December 2020 /

Published online: 2 August 2021

(C) The Author(s) 2021

\begin{abstract}
We consider the construction of semi-implicit linear multistep methods that can be applied to time-dependent PDEs where the separation of scales in additive form, typically used in implicit-explicit (IMEX) methods, is not possible. As shown in Boscarino et al. (J. Sci. Comput. 68: 975-1001, 2016) for Runge-Kutta methods, these semi-implicit techniques give a great flexibility, and allow, in many cases, the construction of simple linearly implicit schemes with no need of iterative solvers. In this work, we develop a general setting for the construction of high order semi-implicit linear multistep methods and analyze their stability properties for a prototype linear advection-diffusion equation and in the setting of strong stability preserving (SSP) methods. Our findings are demonstrated on several examples, including nonlinear reaction-diffusion and convection-diffusion problems.
\end{abstract}

Keywords Semi-implicit methods · Implicit-explicit methods $\cdot$ Multistep methods $\cdot$ Strong stability preserving $\cdot$ High order accuracy

Mathematics Subject Classification 65L06 $\cdot 65 \mathrm{M} 06 \cdot 65 \mathrm{M} 12$

\section{Introduction}

In many applications we are dealing with dynamical systems arising from the spatial discretization (or more generally from the discretization in the phase space) of time dependent partial differential equations. For problems where the various terms have different time scales that can be easily separated the resulting system of ordinary differential equations takes the form

Giacomo Albi

giacomo.albi@univr.it

Lorenzo Pareschi

lorenzo.pareschi@unife.it

1 Computer Science Department, University of Verona, Verona 37134, Italy

2 Mathematics and Computer Science Department, University of Ferrara, Ferrara 44121, Italy 


$$
\frac{\mathrm{d} u(t)}{\mathrm{d} t}=f(t, u(t))+\frac{1}{\varepsilon} g(t, u(t))
$$

with $\varepsilon>0$ a small parameter emphasizing the stiffness in the system. We refer to problem (A) as additive type problem. In (A) the solution $u(t)$ is a vector in $\mathbb{R}^{m}$ which initially satisfies the condition $u(0)=u_{0}$. Typically, the term $f$ contains some nonlinearity or complexity that we do not want to integrate implicitly, whereas the term $g / \varepsilon$ is stiff and requires an implicit integration. For systems of the type (A) implicit-explicit (IMEX) schemes are nowadays a very popular choice $[5,6,13,27]$.

However, in some cases, this separation is not possible and more in general we have a dynamical system of the form

$$
\frac{\mathrm{d} u(t)}{\mathrm{d} t}=\mathcal{H}\left(t, u(t), \frac{u(t)}{\varepsilon}\right),
$$

where the right hand side has a stiff dependence only on the last argument. We refer to problem (G) as generalized partitioned form. Similarly to (A) for problem (G) it is highly desirable to construct a numerical method based on evaluating implicitly only the stiff component $u(t) / \varepsilon$ by keeping explicit the non-stiff one in order to reduce the computational complexity of a fully implicit solver. Following [9] we shall call them semi-implicit methods, since they can be used in a more general context than IMEX methods.

A simple first order method which realizes the above idea reads as follows:

$$
u^{n+1}=u^{n}+\Delta t \mathcal{H}\left(t^{n+1}, u^{n}, \frac{u^{n+1}}{\varepsilon}\right) .
$$

There are many circumstances in which this semi-implicit approach leads to considerable advantages, for example if the function $\mathcal{H}$ is linear with respect to the stiff component the numerical solution can be computed solving only linear systems of equations [8].

The extension of this simple idea to higher order, however, is not straightforward. Here, following the approach recently introduced in [9] for Runge-Kutta methods we construct high order semi-implicit discretization based on the use of linear multistep methods. To this aim, by setting $v(t)=u(t) / \varepsilon$ in $(\mathrm{G})$ we obtain the equivalent formulation for a partitioned system

$$
\left\{\begin{aligned}
\frac{\mathrm{d} u(t)}{\mathrm{d} t} & =\mathcal{H}(t, u(t), v(t)), \\
\varepsilon \frac{\mathrm{d} v(t)}{\mathrm{d} t} & =\mathcal{H}(t, u(t), v(t)),
\end{aligned}\right.
$$

where $v(0)=u_{0} / \varepsilon$. Thus, the formal equivalence among $(\mathrm{G})$ and $(\mathrm{P})$ allows us to adopt IMEX techniques for partitioned systems to more general cases [9, 10]. We refer to [9] for a detailed discussion and results on the equivalence between the various forms of systems (A), (G) and (P), which are usually treated with IMEX methods. For IMEX methods based on Runge-Kutta schemes we refer to [5, 13] as general references. A large literature in this direction has been devoted to the construction of IMEX Runge-Kutta schemes satisfying the asymptotic-preserving (AP) property in the case of hyperbolic problems $[10,12,24$, $27]$ and for kinetic equations $[11,14,16,17]$. For the case of IMEX linear multistep methods, we refer to $[1,2,4,6,18,20,21,30,31]$ for results on the construction and properties 
of the schemes for various types of PDEs and to $[3,15]$ for the construction of schemes satisfying the AP property.

The rest of the manuscript is organized as follows. In the next section, we introduce the IMEX multistep methods for partitioned systems and derive the corresponding semiimplicit formulation for problem (G). Next in Sect. 3 we detail the derivation of the schemes and analyze their stability properties for a prototype linear advection-diffusion equation and in the setting of strong stability preserving (SSP) methods. Section 4 is then devoted to present several numerical applications that confirm the validity of the present approach. The manuscript ends with some conclusions in Sect. 5.

\section{Semi-implicit Multistep Methods}

In this section, we first introduce the general class of IMEX linear multistep schemes for partitioned systems together with some preliminary definitions. Next, we recall some general results on the order conditions and, subsequently, we apply the schemes to the system in the general form $(\mathrm{G})$ to derive the corresponding semi-implicit formulation.

\subsection{IMEX Linear Multistep Methods for Partitioned Systems}

Let us consider a general partitioned system in the form

$$
\left\{\begin{array}{l}
\frac{\mathrm{d} y(t)}{\mathrm{d} t}=\mathcal{F}(t, y(t), z(t)), \\
\frac{\mathrm{d} z(t)}{\mathrm{d} t}=\mathcal{G}(t, y(t), z(t)),
\end{array}\right.
$$

where $y(t) \in \mathbb{R}^{p}$ and $z(t) \in \mathbb{R}^{q}, p, q \geqslant 1$ and $y(0)=y_{0}, z(0)=z_{0}$.

For the partitioned system in the form (2) we consider schemes based on solving the first component with an explicit linear multistep method and the second with an implicit one

$$
\left\{\begin{array}{l}
y^{n+1}=-\sum_{j=0}^{s-1} \tilde{a}_{j} y^{n-j}+\Delta t \sum_{j=0}^{s-1} \tilde{b}_{j} \mathcal{F}\left(t^{n-j}, y^{n-j}, z^{n-j}\right), \\
z^{n+1}=-\sum_{j=0}^{s-1} a_{j} z^{n-j}+\Delta t \sum_{j=-1}^{s-1} b_{j} \mathcal{G}\left(t^{n-j}, y^{n-j}, z^{n-j}\right),
\end{array}\right.
$$

where $b_{-1} \neq 0$. Implicit methods for which $b_{j}=0, j=0, \cdots, s-1$ are referred to as backward differentiation formula (BDF). Another important class is represented by Adams methods, for which $\tilde{a}_{0}=-1, a_{0}=-1, \tilde{a}_{j}=0, a_{j}=0, j=1, \cdots, s-1$.

Remark 1 Classical systems in additive form (A), i.e.,

$$
\frac{\mathrm{d} u(t)}{\mathrm{d} t}=f(t, u(t))+\frac{1}{\varepsilon} g(t, u(t))
$$


with initial data $u(0)=u_{0}$ can be also written in partitioned form by defining $u(t)=y(t)+z(t), \mathcal{F}(t, y(t), z(t))=f(t, u(t)), \mathcal{G}(t, y(t), z(t))=g(t, u(t)) / \varepsilon$ and rewriting

$$
\left\{\begin{array}{l}
\frac{\mathrm{d} y(t)}{\mathrm{d} t}=\mathcal{F}(t, y(t), z(t)), \\
\frac{\mathrm{d} z(t)}{\mathrm{d} t}=\mathcal{G}(t, y(t), z(t))
\end{array}\right.
$$

for any initial data such that $y(0)+z(0)=u_{0}$.

\subsection{Semi-implicit Methods in Predictor-Corrector Form}

Let us now apply the previous general formulation to the case of system $(\mathrm{G})$ in the partitioned form $(\mathrm{P})$, i.e.,

$$
\left\{\begin{array}{l}
\frac{\mathrm{d} u(t)}{\mathrm{d} t}=\mathcal{H}(t, u(t), v(t)), \\
\frac{\mathrm{d} v(t)}{\mathrm{d} t}=\mathcal{H}(t, u(t), v(t)),
\end{array}\right.
$$

where in order to simplify notations, we remove the dependence on the parameter $\varepsilon$ in the second argument, keeping in mind that this dependence is stiff.

We obtain the semi-implicit multistep solver

$$
\left\{\begin{array}{l}
u^{n+1}=-\sum_{j=0}^{s-1} \tilde{a}_{j} u^{n-j}+\Delta t \sum_{j=0}^{s-1} \tilde{b}_{j} \mathcal{H}\left(t^{n-j}, u^{n-j}, v^{n-j}\right), \\
v^{n+1}=-\sum_{j=0}^{s-1} a_{j} v^{n-j}+\Delta t \sum_{j=-1}^{s-1} b_{j} \mathcal{H}\left(t^{n-j}, u^{n-j}, v^{n-j}\right),
\end{array}\right.
$$

where initially we assume $v^{n-j}=u^{n-j}, j=0, \cdots, s-1$. Let us point out that even if the scheme doubles the number of unknown the number of evaluations of the function $\mathcal{H}(t, u(t), v(t))$ is not doubled since both schemes use the same time levels. In particular, if for notation simplicity we assume the system to be autonomous and the function $\mathcal{H}$ to depend linearly from the second argument

$$
\mathcal{H}(u(t), v(t))=\mathcal{K}(u(t))+A(u(t)) v(t),
$$

where the function $\mathcal{K}: \mathbb{R}^{m} \rightarrow \mathbb{R}^{m}$ and $A(u(t))$ is an invertible $m \times m$ matrix, the resulting scheme can be solved without any need of an iterative solver. In fact, the second equation in (7) can be rewritten as

$$
\begin{aligned}
v^{n+1}= & -\sum_{j=0}^{s-1} a_{j} v^{n-j}+\Delta t \sum_{j=0}^{s-1} b_{j} \mathcal{H}\left(u^{n-j}, v^{n-j}\right) \\
& +\Delta t b_{-1}\left(\mathcal{K}\left(u^{n+1}\right)+A\left(u^{n+1}\right) v^{n+1}\right),
\end{aligned}
$$

or equivalently in explicit form 


$$
\begin{aligned}
v^{n+1}= & \left(I-b_{-1} \Delta t A\left(u^{n+1}\right)\right)^{-1}\left(-\sum_{j=0}^{s-1} a_{j} v^{n-j}+\Delta t \sum_{j=0}^{s-1} b_{j} \mathcal{H}\left(u^{n-j}, v^{n-j}\right)\right. \\
& \left.+\Delta t b_{-1}\left(\mathcal{K}\left(u^{n+1}\right)\right)\right)
\end{aligned}
$$

since $u^{n+1}$ is computed from the first equation in (7).

For semi-implicit Runge-Kutta methods (see [9]) in the case of autonomous systems it is possible to construct the scheme in such a way that the two solutions provided by the system at time $n+1$ coincide. Otherwise for scheme (7) at time level $n+1$ we will have two distinct numerical solutions $u^{n+1}$ and $v^{n+1}$ approximations of the true solution $u\left(t^{n+1}\right)$ of problem $(\mathrm{G})$. Note, however, that both these solutions are used by the scheme to advance in time.

To define a unique solution, it is natural to consider the scheme (7) as a predictorcorrector multistep method for the non-stiff component, where the explicit scheme is used to predict $u^{n+1}$ which is then used by the implicit solver as a corrector for $v^{n+1}$.

As an example, reverting back to the notations used initially, we can write the semiimplicit scheme for $(\mathrm{G})$ as

$$
\left\{\begin{aligned}
\hat{u}^{n+1}= & -\sum_{j=0}^{s-1} \tilde{a}_{j} u^{n-j}+\Delta t \sum_{j=0}^{s-1} \tilde{b}_{j} \mathcal{H}\left(t^{n-j}, u^{n-j}, \frac{u^{n-j}}{\varepsilon}\right) \\
u^{n+1}= & -\sum_{j=0}^{s-1} a_{j} u^{n-j}+\Delta t \sum_{j=0}^{s-1} b_{j} \mathcal{H}\left(t^{n-j}, u^{n-j}, \frac{u^{n-j}}{\varepsilon}\right) \\
& +\Delta t b_{-1} \mathcal{H}\left(t^{n+1}, \hat{u}^{n+1}, \frac{u^{n+1}}{\varepsilon}\right) .
\end{aligned}\right.
$$

The above scheme uniquely identifies the numerical solution at time $u^{n+1}$. In the following section we will discuss the general order conditions for (3) and present different types of semi-implicit multistep methods of various order.

Remark 2 As a consequence of the above predictor-corrector formulation for the non-stiff component, if the implicit solver has order $p$ it is typically enough to consider an explicit solver of order $p-1$. We emphasize that this predictor-corrector interpretation holds true only for the non-stiff component, since the stiff one is treated implicitly by the resulting scheme.

\section{Order Conditions, Stability and Derivation of the Schemes}

In this section, we provide the details of the schemes that will be used in our numerical results. First, we recall the order condition and then some general definitions concerning the stability properties. The stability properties for IMEX multistep methods are usually discussed in the case of additive systems for simple one-dimensional convection-diffusion problems $[6,18,20,21]$. We also refer to the recent stability analysis in [3] for the case of two dimensional partitioned systems. 


\subsection{Order Conditions}

For a partitioned system in the form (3) an order $p$ scheme is obtained if both schemes are of order $p$, namely the following conditions are satisfied:

$$
\left\{\begin{array}{l}
1+\sum_{j=0}^{s-1} \tilde{a}_{j}=0, \quad 1+\sum_{j=0}^{s-1} a_{j}=0 \\
1-\sum_{j=1}^{s-1} j \tilde{a}_{j}=\sum_{j=0}^{s-1} \tilde{b}_{j}, \quad 1-\sum_{j=1}^{s-1} j a_{j}=\sum_{j=-1}^{s-1} b_{j}, \\
\frac{1}{2}+\sum_{j=1}^{s-1} \frac{j^{2}}{2} \tilde{a}_{j}=-\sum_{j=1}^{s-1} \tilde{b}_{j}, \quad \frac{1}{2}+\sum_{j=1}^{s-1} \frac{j^{2}}{2} a_{j}=b_{-1}-\sum_{j=1}^{s-1} j b_{j} \\
\vdots \quad \vdots \quad \frac{1}{p !}+\sum_{j=1}^{s-1} \frac{(-j)^{p}}{p !} a_{j} \\
=\sum_{j=1}^{s-1} \frac{(-j)^{p-1}}{(p-1) !} \tilde{b}_{j}, \quad=\frac{b_{-1}}{(p-1) !}+\sum_{j=1}^{s-1} \frac{(-j)^{p-1}}{(p-1) !} b_{j} .
\end{array}\right.
$$

We recall that an $s$-step implicit multistep scheme can achieve order $s+1$, while an $s$-step explicit method has only order at most $s$. We refer to $[6,18,20,23]$ for more details on the order conditions for IMEX multistep schemes in the case of additive systems. Here, we remark that the order conditions for the partitioned scheme are simpler than in the case of additive schemes where there is a coupling between the explicit and the implicit solver. In addition, in the case of system (9) only $p-1$ order of accuracy is required by the explicit predictor solver to guarantee that an order $p$ implicit corrector step yields the desired order $p$ accuracy.

\subsection{Stability Properties}

The stability properties are usually analyzed for simple one-dimensional linear problems of the form

$$
\frac{\mathrm{d} u(t)}{\mathrm{d} t}=\mathrm{i} \lambda u(t)+\mu u(t)
$$

where $\lambda, \mu \in \mathbb{R}$ and $\mathrm{i}$ is the imaginary unit. These kinds of problems typically are originated by the space discretization of convection diffusion equations, hyperbolic balance laws or linear kinetic models $[6,15,18,20,23]$. For example, in the case of one-dimensional linear convection diffusion problems

$$
\frac{\partial u}{\partial t}=a \frac{\partial u}{\partial x}+D \frac{\partial^{2} u}{\partial x^{2}}
$$

discretized by standard central differences of the mesh $\Delta x$ we have (11) with 


$$
\lambda=\frac{a}{\Delta x} \sin (k \Delta x), \quad \mu=\frac{2 D}{(\Delta x)^{2}}(\cos (k \Delta x)-1),
$$

where $k$ is the frequency of the corresponding Fourier mode.

In partitioned form system (11) will correspond to system

$$
\left\{\begin{array}{l}
\frac{\mathrm{d} u(t)}{\mathrm{d} t}=\mathrm{i} \lambda u(t)+\mu v(t), \\
\frac{\mathrm{d} v(t)}{\mathrm{d} t}=\mathrm{i} \lambda u(t)+\mu v(t) .
\end{array}\right.
$$

Note that, since the direct application of an IMEX multistep method to a system in the additive form (11) is not equivalent to the application of the combination of an explicit and an implicit multistep scheme to the partitioned form (12) even the resulting stability analysis will differ.

Applying the semi-implicit scheme (7)-(12) yields

$$
\left\{\begin{aligned}
u^{n+1}= & -\sum_{j=0}^{s-1} \tilde{a}_{j} u^{n-j}+\Delta t \sum_{j=0}^{s-1} \tilde{b}_{j}\left(\mathrm{i} \lambda u^{n-j}+\mu v^{n-j}\right) \\
v^{n+1}= & \left(\frac{1}{1-\mu b_{-1} \Delta t}\right)\left(-\sum_{j=0}^{s-1} a_{j} v^{n-j}+\Delta t \sum_{j=0}^{s-1} b_{j}\left(\mathrm{i} \lambda u^{n-j}+\mu v^{n-j}\right)\right. \\
& \left.+\Delta t b_{-1} \mathrm{i} \lambda u^{n+1}\right)
\end{aligned}\right.
$$

where $u^{n-j}=v^{n-j}, j=0, \cdots, s-1$. By direct substitution of the first equation into the second we obtain the explicit form

$$
\begin{aligned}
v^{n+1}= & \left(\frac{1}{1-\mu b_{-1} \Delta t}\right)\left(-\sum_{j=0}^{s-1} a_{j} v^{n-j}+\Delta t \sum_{j=0}^{s-1} b_{j}\left(\mathrm{i} \lambda v^{n-j}+\mu v^{n-j}\right)\right. \\
& \left.+\Delta t b_{-1} \mathrm{i} \lambda\left(-\sum_{j=0}^{s-1} \tilde{a}_{j} v^{n-j}+\Delta t \sum_{j=0}^{s-1} \tilde{b}_{j}\left(\mathrm{i} \lambda v^{n-j}+\mu v^{n-j}\right)\right)\right) .
\end{aligned}
$$

This leads to the characteristic equation

$$
\zeta^{s}\left(1-b_{-1} z_{R}\right)+\rho(\zeta)-\left(z_{R}+z_{I}\right) \sigma(\zeta)+b_{-1} z_{I}\left(\tilde{\rho}(\zeta)-\left(z_{R}+z_{I}\right) \tilde{\sigma}(\zeta)\right)=0
$$

where $z_{R}=\mu \Delta t, z_{I}=\mathrm{i} \lambda \Delta t$ and

$$
\begin{array}{ll}
\rho(\zeta)=\sum_{j=0}^{s-1} a_{j} \zeta^{s-j-1}, & \sigma(\zeta)=\sum_{j=0}^{s-1} b_{j} \zeta^{s-j-1}, \\
\tilde{\rho}(\zeta)=\sum_{j=0}^{s-1} \tilde{a}_{j} \zeta^{s-j-1}, & \tilde{\sigma}(\zeta)=\sum_{j=0}^{s-1} \tilde{b}_{j} \zeta^{s-j-1} .
\end{array}
$$

Stability corresponds to the requirement that all roots have modulus less than or equal one and that all multiple roots have modulus less than one. 


\subsection{Derivation of the Schemes}

In the sequel, to simplify the notation, we restrict to autonomous systems (this is always possible simply by augmenting the dimension of the system by one), i.e., the function $\mathcal{H}$ does not depend explicitly on time. Let us first point out that the simplest first-order method (1) is common to multistep methods and Runge-Kutta methods and in the case of system (6) reads

$$
\begin{aligned}
& u^{n+1}=u^{n}, \\
& v^{n+1}=v^{n}+\Delta t \mathcal{H}\left(u^{n+1}, v^{n+1}\right)
\end{aligned}
$$

with $v^{n}=u^{n}$, which corresponds to a simple identity as explicit predictor for the non-stiff component and backward Euler as implicit corrector for the stiff one.

Second-order methods The general form of second order schemes for (6) reads as follows:

$$
\begin{aligned}
u^{n+1}= & u^{n}+\Delta t \mathcal{H}\left(u^{n}, v^{n}\right), \\
v^{n+1}= & \frac{1}{2 \alpha+1}\left(4 \alpha u^{n}-(2 \alpha-1) u^{n-1}\right)+\frac{\Delta t}{2 \alpha+1}\left((2 \alpha+\beta) \mathcal{H}\left(u^{n+1}, v^{n+1}\right)\right. \\
& \left.+2(1-\alpha-\beta) \mathcal{H}\left(u^{n}, v^{n}\right)+\beta \mathcal{H}\left(u^{n-1}, v^{n-1}\right)\right)
\end{aligned}
$$

with $u^{n}=v^{n}, u^{n-1}=v^{n-1}$ and the solver for the non-stiff component is represented by the forward Euler. Popular choices for the implicit solver are obtained for $\alpha=1 / 2$ and $\beta=0$ which corresponds to Crank-Nicholson, the resulting scheme will be referred to as FE-CN2, and $\alpha=1$ and $\beta=0$ corresponding to the second order BDF scheme, we will refer to this scheme as FE-BDF2. In the case of $\alpha=1 / 2$ the value of $\beta=1 / 8$ yields the best damping properties [6], the resulting scheme requires the additional storage of level $n-1$ and is referred to as FE-MCN2.

Third-order methods and higher The most natural way to obtain third-order methods, as a combination of an explicit and an implicit multistep method, is to use a two-step Adams-Bashforth method with a two-step Adams-Moulton method. This same strategy actually can be used to obtain methods of a higher order. We will refer to this general class of schemes as AB-AM $p$, where $p$ is the order of the resulting scheme. Except for AB-AM2, which is the same as FE-CN2, these schemes in general suffer from poor stability properties when $\mu \ll 0$ (see Fig. 1). Replacing the Adams-Moulton methods with BDF schemes with the same order yields a class of schemes with better stability properties referred to as AB-BDFp, where $p$ is the order of the resulting scheme. In this way, $\mathrm{AB}-\mathrm{BDF} 2$ is the same as FE-BDF2.

We report in Fig. 2 the stability contours for various semi-implicit multistep methods up to order four.

Strong stability preserving methods Often the time integration of PDEs requires some monotonicity properties to be satisfied. An important class of methods in this direction is represented by the so-called strong stability preserving (SSP) methods. These methods were designed specifically for solving the ODEs coming from a semi-discrete, spatial discretization of time dependent partial differential equations, especially hyperbolic PDEs and convection-diffusion problems [19]. In summary, these schemes are stable for a certain (semi) norm 

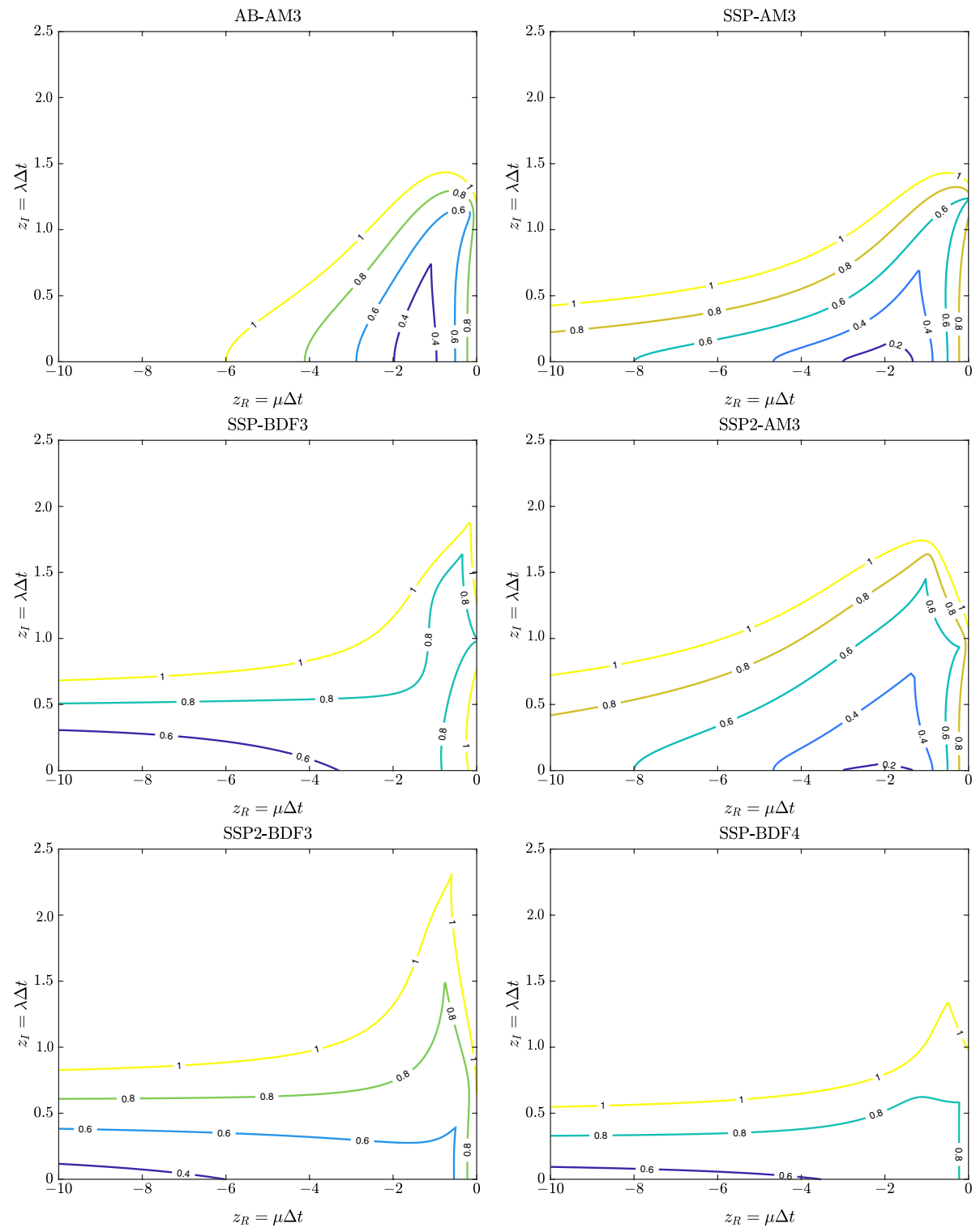

Fig. 1 Contours of the stability region in the case of problem (11) for third-order and fourth-order SSP methods. The use of the SSP property permits to improve the stability behavior for $\mu \ll 0$

$$
\left\|u^{n+1}\right\| \leqslant\left\|u^{n}\right\|
$$

under a suitable time step restriction $\Delta t \leqslant \Delta t_{0}$. Typically these schemes are applied to the convective part and $\Delta t_{0}$ refers to the stability constraint, usually referred to as CFL condition, which links $\Delta t_{0}=C \Delta t_{\mathrm{FE}}$, where $C$ is the CFL coefficient and $\Delta t_{\mathrm{FE}}$ the stability constraint of the SSP property in the forward Euler scheme. 

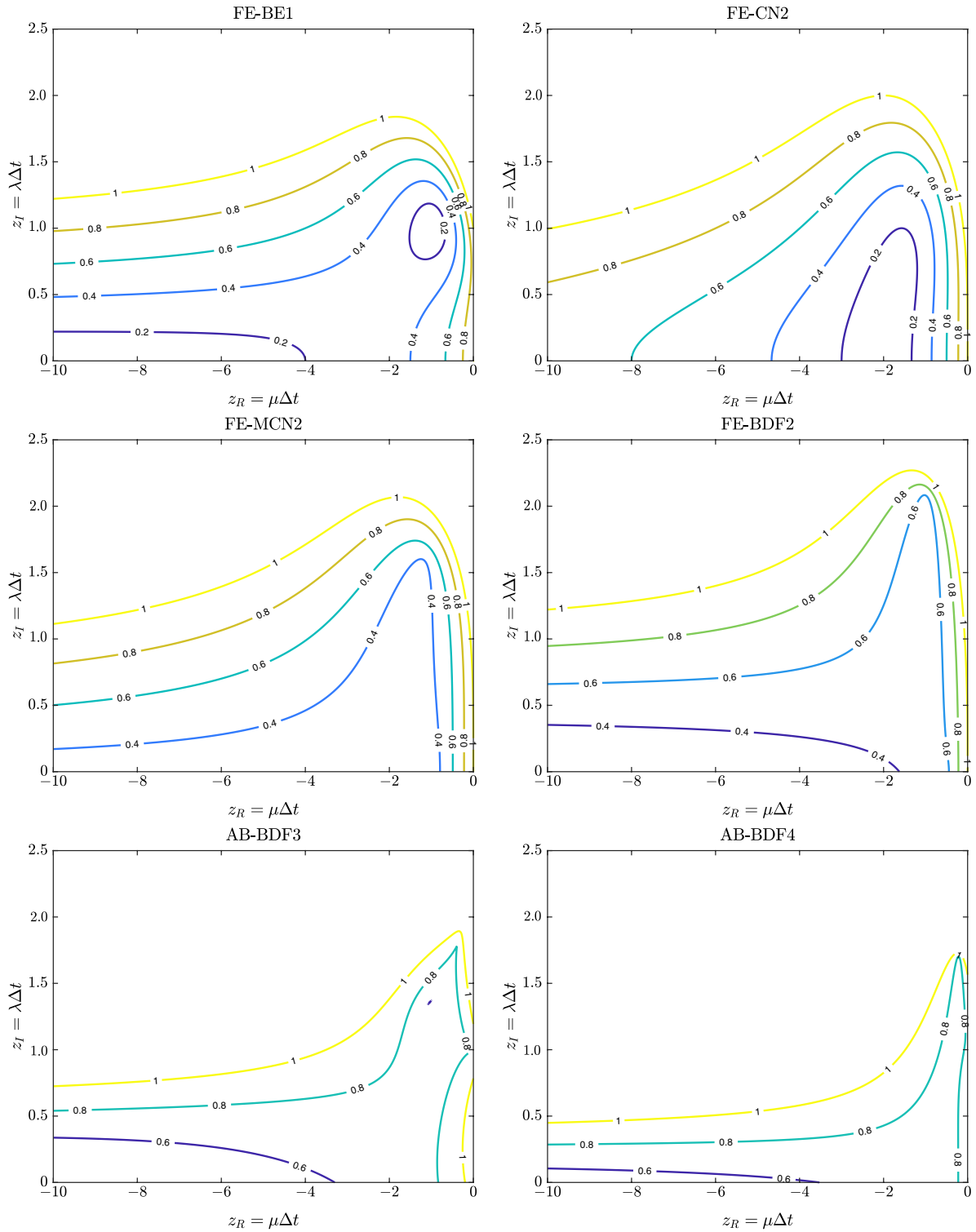

Fig. 2 Contours of the stability region in the case of problem (11) for some semi-implicit multistep methods of order one to four. Increasing the order of accuracy the stability constraints on $\Delta t$ become more severe for $\mu \ll 0$

In [19] it is shown that there are no implicit multistep SSP schemes of order higher than 1. Therefore, we can combine optimal explicit multistep methods satisfying the SSP property (14) as a predictor for the non-stiff component with implicit methods to improve the overall stability region in our semi-implicit schemes. 
The explicit multistep SSP schemes are of the general form

$$
u^{n+1}=\sum_{j=0}^{s-1}\left(\alpha_{j} u^{n-j}+\Delta t \beta_{j} \mathcal{H}\left(u^{n-j}, v^{n-j}\right)\right), \quad \alpha_{j} \geqslant 0 .
$$

The optimal second order two steps explicit SSP method $(C=1 / 2)$ corresponds to the choices $\boldsymbol{\alpha}=(4 / 5,1 / 5)^{\mathrm{T}}$ and $\boldsymbol{\beta}=(8 / 5,-2 / 5)^{\mathrm{T}}$, whereas the optimal third order explicit four steps scheme $(C=1 / 3)$ is obtained for $\alpha=(16 / 27,0,0,11 / 27)^{\mathrm{T}}$ and $\boldsymbol{\beta}=(16 / 9,0,0,4 / 9)^{\mathrm{T}}$ (see [19]). The corresponding schemes are denoted as SSP-AM3, SSP-BDF3 and SSP-BDF4. If one increases the number of steps, then SSP methods can be found to have larger SSP regions. Because there is no significant increase in the computational cost when the number of steps is increased, if storage is not a consideration, it may be advantageous to use an SSP multi-step methods with more steps and larger stability domain. For example, the optimal second order four steps SSP method $(C=2 / 3)$ is obtained with $\boldsymbol{\alpha}=(8 / 9,0,0,1 / 9)^{\mathrm{T}}$ and $\boldsymbol{\beta}=(4 / 3,0,0,0)^{\mathrm{T}}$. The corresponding third order semi-implicit schemes are denoted as SSP2-AM3 and SSP2-BDF3.

We report in Fig. 1, the stability contours of AB-AM3 and SSP-AM3. The importance of the optimal SSP property in the explicit scheme is evident and improves dramatically the stability properties of the resulting method. For large values of $|\mu|$ the semi-implicit method based on AM3 becomes stable under a reasonable CFL condition of $\Delta t z_{I} \leqslant 1 / 2$. Similarly the use of SSP2 predictor increases this stability constraint to approximatively $\Delta t z_{I} \leqslant 3 / 5$. We also give in the same figure the optimal third- and fourth-order methods constructed using the BDF formulae. Again the use of SSP2 predictor slightly improves the stability properties for large $|\mu|$.

\section{Numerical Results}

In what follows we investigate semi-implicit schemes for non-linear problems with stiff terms, where the stiffness can be solved efficiently by linear solvers.

We will discuss non-linear diffusion-reaction and convective-diffusion problems, following different examples extracted from $[9,22]$.

\subsection{Test 1: Order of Convergence for Reaction-Diffusion System}

Following the validation example of [9] we consider the non-autonomous diffusion-reaction system, where $\omega=\left(\omega_{1}, \omega_{2}\right): \mathbb{R}_{+} \times[0,2 \pi)^{2} \rightarrow \mathbb{R}^{2}$ is solution of the following system

$$
\left\{\begin{array}{l}
\partial_{t} \omega_{1}=\Delta \omega_{1}-\alpha(t) \omega_{1}^{2}+\frac{9}{2} \omega_{1}+\omega_{2}+f(t), \\
\partial_{t} \omega_{2}=\Delta \omega_{2}+\frac{7}{2} \omega_{2}
\end{array}\right.
$$

and the time dependent factors are $\alpha(t)=2 \mathrm{e}^{t / 2}$ and $f(t)=-2 \mathrm{e}^{-t / 2}$. Accounting periodic boundary conditions, the initial data is extracted from the exact solution

$$
\left\{\begin{array}{l}
\omega_{1}(t, x, y)=\mathrm{e}^{-t / 2}(1+\cos (x)), \\
\omega_{2}(t, x, y)=\mathrm{e}^{-t / 2} \cos (2 x),
\end{array} \quad(x, y) \in[0,2 \pi)^{2} .\right.
$$


To apply the semi-implicit multistep scheme (7) we reformulate system (16) introducing $u=\left(u_{1}, u_{2}\right)$ and $v=\left(v_{1}, v_{2}\right)$, and the operator

$$
\mathcal{H}(t, u, v)=\left(\begin{array}{c}
\Delta v_{1}-\alpha(t) u_{1} v_{1}+\frac{9}{2} v_{1}+v_{2}+f(t) \\
\Delta v_{2}+\frac{7}{2} v_{2}
\end{array}\right) .
$$

We initialize the multistep scheme evaluating the exact solution for the first steps.

For the spatial discretization of the diffusion operator we apply a sixth order central finite difference, on a uniform grid for the periodic domain $[0,2 \pi)^{2}$ with $\Delta x=\Delta y$. For $u(t, x, y)$ evaluated on a point $\left(t_{n}, x_{i}, y_{j}\right)$ we consider the operators

$$
\left(D_{x x} u^{n}\right)_{i j}=\frac{2 u_{i+3 j}^{n}-27 u_{i+2 j}^{n}+270 u_{i+1 j}^{n}-240 u_{i j}^{n}+270 u_{i-1 j}^{n}-27 u_{i-2 j}^{n}+2 u_{i-3 j}^{n}}{180 \Delta x^{2}},
$$

and similarly $D_{y y}$ for the $y$-direction, taking into account periodic boundary conditions.

To estimate the order of accuracy in time we proceed refining the space step and time steps with stability conditions

$$
\Delta t=\lambda \Delta x,
$$

where $\Delta x=\Delta y$ and $\lambda$ to be chosen according to the stability property of the scheme. Thus we refine simultaneously the space step and the time step and we monitor the $\ell_{\infty}$ norm of the error decay for the numerical solution $\omega_{i j}(t)$ at final time $T=2$

$$
\varepsilon_{\infty}\left(\omega^{(k)}\right)=\max _{i, j}\left\|\omega_{i j}^{(k)}(T)-\omega\left(x_{i}, y_{j}, T\right)\right\|,
$$

where $\omega_{i j}^{(k)}(T)$ is the numerical solution evaluated on the uniform grid and $\omega\left(x_{i}, y_{j}, t_{n}\right)$ is the exact solution evaluated at $\left(x_{i}, y_{j}, T\right)$.

In Fig. 3 we report the error decay $\varepsilon_{\infty}$ for different schemes and different choice of the CFL parameter. In the left plot, we fix to $\lambda=0.25$ and compare with the results obtained in [9] where $\lambda=0.5$. We observe similar accuracy for schemes of the same order but for a smaller CFL condition, on the other hand, we show that higher order is achievable. In the right plot, we have modified the CFL parameter according to the order of the scheme: we keep $\lambda=0.25$ for fourth order schemes, for third order is increased to $\lambda=0.3$, for second
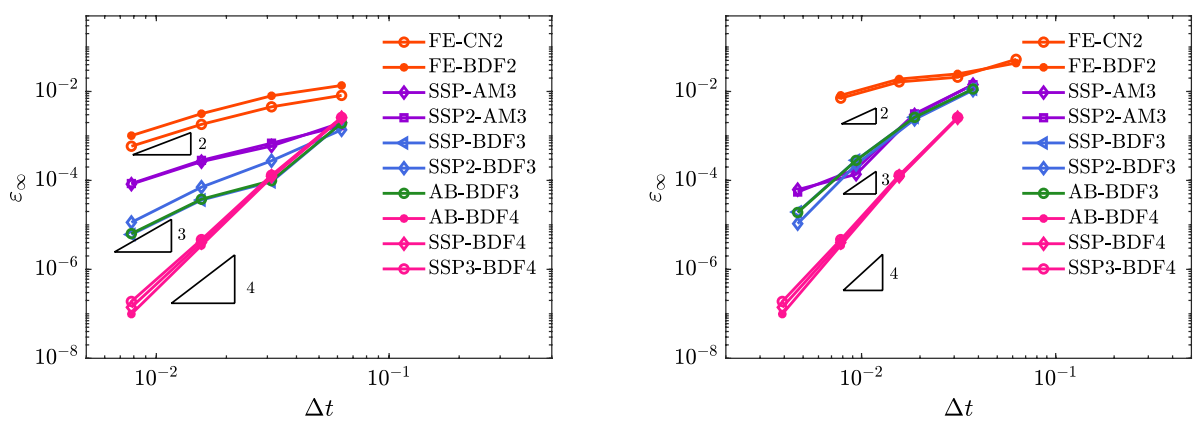

Fig. 3 Test 1: we report the error decay computed according to (18) for the solution of (16) $\omega=\left(\omega_{1}, \omega_{2}\right)$. Left plot reports the $\varepsilon_{\infty}$ error, for $\lambda=0.25$. Right plot depicts different choice of the CFL is adapted: for second order $\lambda=0.5$, for third order $\lambda=0.3$, and for fourth order $\lambda=0.25$ 
order we use $\lambda=0.5$. According to the stability results we observe that for lower-order schemes the CFL condition can be relaxed.

\subsection{Test 2: Non-linear Reaction Diffusion System}

We consider the Gray-Scott model, as studied in [22, 26, 32],

$$
\left\{\begin{array}{l}
\partial_{t} \omega_{1}=\sigma_{1} \Delta \omega_{1}-\omega_{1} \omega_{2}^{2}+\gamma\left(1-\omega_{1}\right), \\
\partial_{t} \omega_{2}=\sigma_{2} \Delta \omega_{2}+\omega_{1} \omega_{2}^{2}-(\gamma+\kappa) \omega_{2},
\end{array}\right.
$$

where $\omega=\left(\omega_{1}, \omega_{2}\right): \mathbb{R}_{+} \times[-1,1)^{2} \rightarrow \mathbb{R}^{2}$ with periodic boundary conditions, and initial data

$$
\left\{\begin{array}{l}
\omega_{1}(0, x, y)=1-2 \omega_{2}(0, x, y), \\
\omega_{2}(0, x, y)=\frac{1}{4} \sin ^{2}(4 \pi x) \sin ^{2}(4 \pi y),
\end{array} \quad(x, y) \in[-1 / 4,1 / 4]^{2},\right.
$$

and zero otherwise on the squared domain $[-1,1]^{2}$. The diffusion and reaction parameters are

$$
\sigma_{1}=8 \times 10^{-5}, \quad \sigma_{2}=4 \times 10^{-5}, \quad \gamma=0.024, \quad \kappa=0.06
$$

Initial data and parameters are selected in order to match the test proposed in [22] and inspired from [26]. In order to apply the semi-implicit multi-step scheme (7) we reformulate system (19) introducing $u=\left(u_{1}, u_{2}\right)$ and $v=\left(v_{1}, v_{2}\right)$, and the operator

$$
\mathcal{H}(t, u, v)=\left(\begin{array}{c}
D_{1} \Delta u_{1}-v_{1} u_{2}^{2}+\gamma\left(1-v_{1}\right) \\
D_{2} \Delta u_{2}+v_{1} u_{2}^{2}-(\gamma+\kappa) v_{2}
\end{array}\right),
$$

where now we treat explicitly the diffusion terms, since $D_{1}, D_{2}$ are negligible with respect to the reaction coefficients.

Thus the linear reaction terms are taken implicitly, whereas the non-linear term is taken implicit only in the $\omega_{1}$ component. We employ multistep scheme SSP3-BDF4 for time integration and fourth order central difference for the space discretization, introducing the operators

$$
\left(D_{x x} u^{n}\right)_{i j}=\frac{-u_{i+2 j}^{n}+16 u_{i+1 j}^{n}-30 u_{i j}^{n}+16 u_{i-1 j}^{n}-u_{i-2 j}^{n}}{12 \Delta x^{2}},
$$

and similarly $D_{y y}$ for the $y$-direction, taking into account periodic boundary conditions. Initialization of the multistep scheme is performed via Matlab solver ode 45.

Figure 4 reports the evolution of the component $\omega_{2}(t, x, y)$ for different times. Starting from a symmetric concentration Gray-Scott model produces spot multiplication, resembling cell division process. The computational domain is discretized with $N_{x}=N_{y}=200$ points in space, final time is $T=1500$ with uniform step $\Delta t=\Delta x / 2$.

We additionally show in Fig. 5 the marginal distributions of the second component computed as

$$
\bar{\omega}_{2}(t, x)=\int_{-10}^{10} \omega_{2}(t, x, y) \mathrm{d} y .
$$



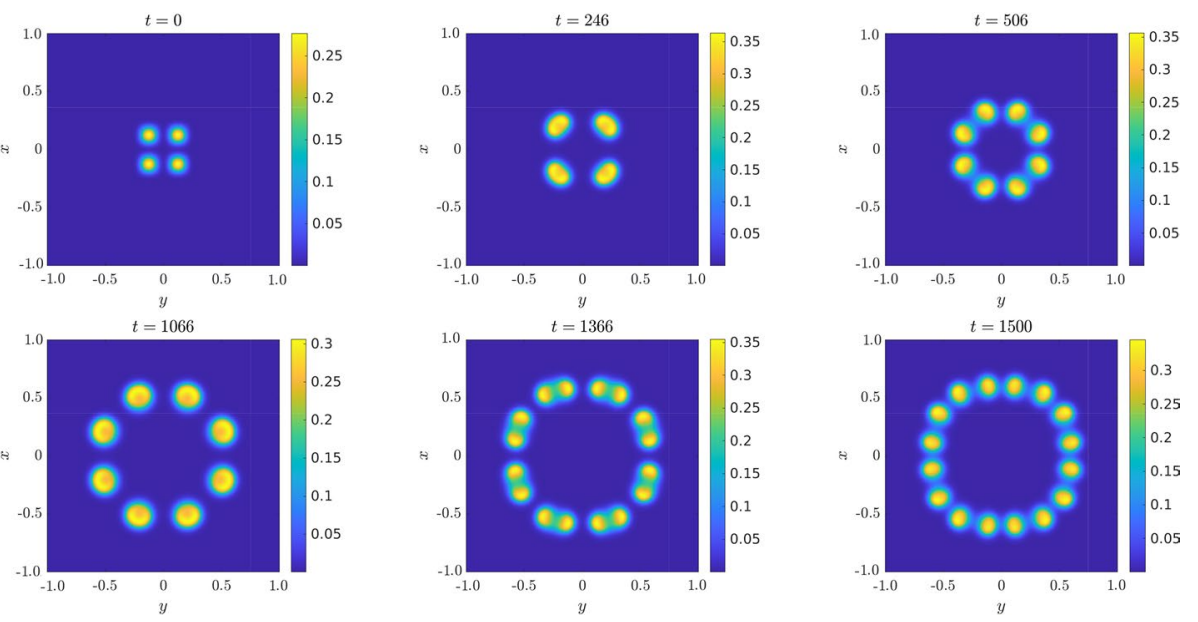

Fig. 4 Test 2: (Gray-Scott model) Solution of the reaction-diffusion system (19) in the component $\omega_{2}$ at different time frames obtained with scheme SSP3-BDF4 on the square $\Omega=[-1,1]^{2}$ with uniform spacetime discretization with $\Delta x=0.01$ and $\Delta t=\Delta x$. Parameters of the model are $\sigma_{1}=8 \times 10^{-5}, \sigma_{2}=4 \times 10^{-5}$, $\gamma=0.024$ and $\kappa=0.06$
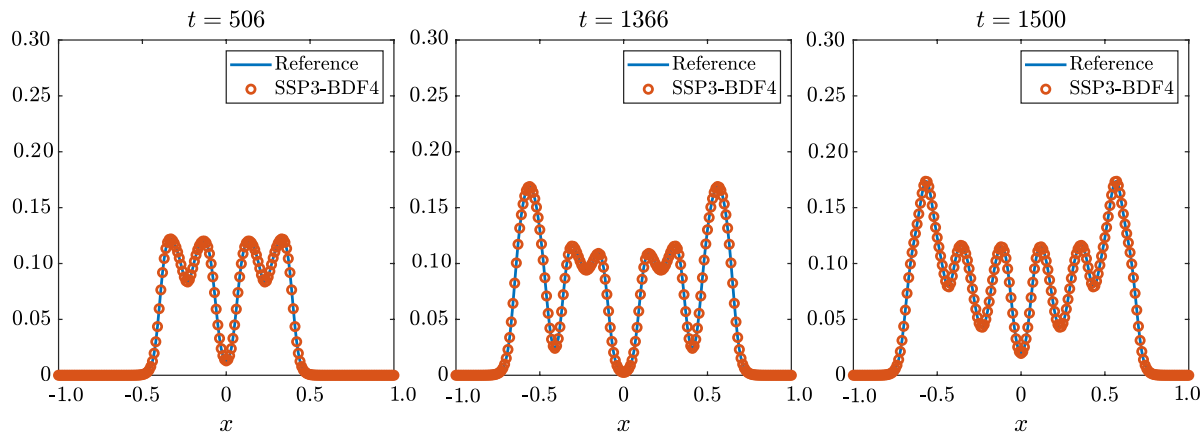

Fig. 5 Test 2: (Gray-Scott model) Marginal distributions of the reaction-diffusion system (19) of the $\omega_{2}$ component computed with SSP3-BDF4 computed with $N_{x}=N_{y}=200$ points in each direction

compared with a reference with $N_{x}=N y=400$ points.

\subsection{Test 3: Nonlinear Convection-Diffusion}

We finally consider the nonlinear convection diffusion model defined on the full plan $x \in \mathbb{R}^{2}$ as follows: 


$$
\left\{\begin{array}{l}
\partial_{t} \omega+(E+\mu \nabla \log (\omega)) \cdot \nabla \omega=\mu \Delta \omega, \\
\omega_{0}(0, x)=\mathrm{e}^{-\|x\|^{2} / 2},
\end{array}\right.
$$

where $E=(1,1)^{\mathrm{T}}$ and $\mu=0.5$. The initial data is extracted from the exact solution given by

$$
\omega(t, x)=\frac{1}{\sqrt{4 \mu t+1}} \mathrm{e}^{-\frac{\|x-E t\|^{2}}{8 \mu t+2}} .
$$

In order to solve numerically (22) we introduce the operator

$$
\mathcal{H}(t, u, v)=-(E+\mu \nabla \log (u)) \cdot \nabla v+\mu \Delta v,
$$

where we treat the convection and diffusion terms implicitly, on the computational domain $[-10,10]$ up to final time $T=1$. We choose the uniform space step $\Delta x=\Delta y=0.1$ and $\Delta t=\Delta x / 2$. For time integration we compare different schemes FE-CN2, SSP2-AM3, and SSP-BDF4. For space we use the 4th order central difference for diffusion operator, as in (21), and for the convective term we account the operator

$$
\left(D_{x} u^{n}\right)_{i j}=\frac{-u_{i+2 j}+8 u_{i+1 j}-8 u_{i-1 j}+u_{i-2 j}}{12 \Delta x},
$$
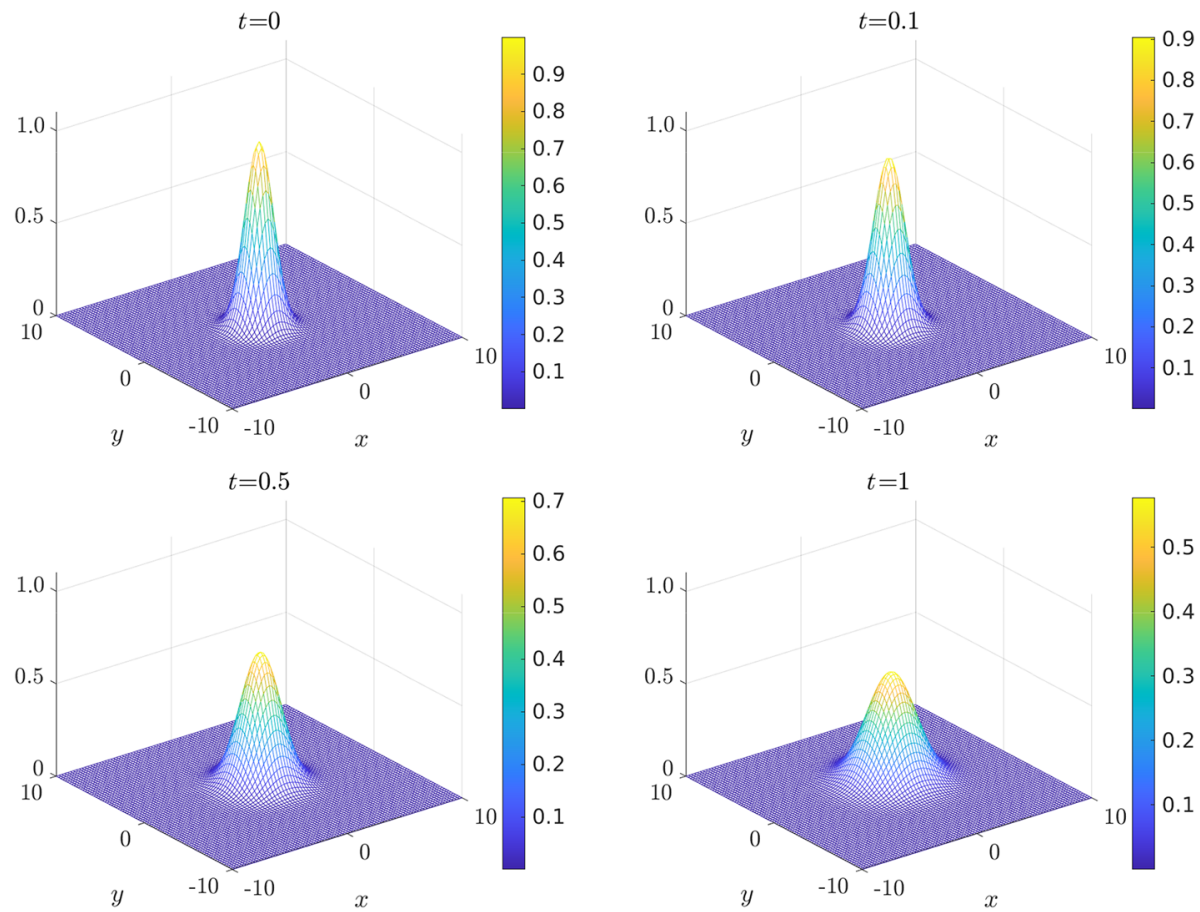

Fig. 6 Test 3: (non-linear convection diffusion). Numerical solution of model (22) on the domain [-10, 10] up to final time $T=1$. Space step $\Delta x=\Delta y=0.1$ and $\Delta t=\Delta x / 2$. Time integration is performed with LM scheme SSP3-BDF4 and 4th order central difference for convective and diffusion operator 
Fig. 7 Test 3: (nonlinear convection-diffusion). Marginal distribution of system (22). The numerical solution is computed using different schemes: FE-CN2, SSP2-AM3, SSP-BDF4 with $N x=N y=100$ points and $\Delta t=\lambda \Delta x$ with $\lambda=0.5$. We observe that increasing higher order method is able to capture better profile of the reference solution

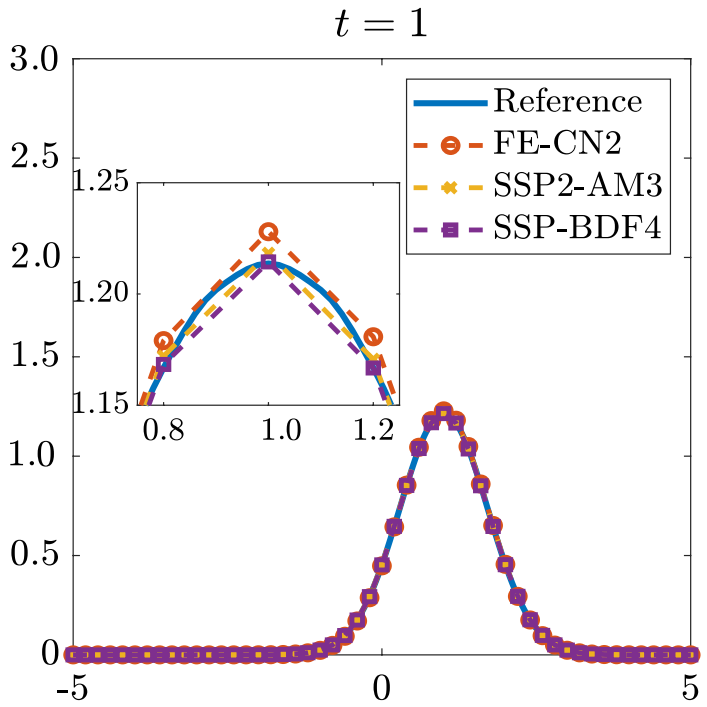

and equivalently $D_{y}$ for the $y$-direction. In Fig. 6 we report the numerical solution at different times from $t=0$ to $t=1$.

In Fig. 7 we present the marginal distribution of the solution $\omega(x, y)$. We observe that increasing order of the semi-implicit multistep has a better coherence with the profile of the reference solution.

\section{Conclusions}

We derived high order semi-implicit schemes based on linear multistep methods. The schemes have been constructed following the approach recently introduced in [9] for Runge-Kutta methods. The resulting time discretizations have a predictor-corrector structure and, compared with Runge-Kutta methods, do not require additional order conditions so that they can easily reach high order accuracy. Numerical tests for schemes up to fourth-order accuracy have been presented in the case of nonlinear reaction-diffusion and convection-diffusion problems. From a practical point of view it has been observed that order three and four schemes represent the best compromise between accuracy and stability (and, therefore, computational cost) while higher order schemes have more severe stability restrictions. For convection dominated problems, the use of multistep predictor methods that satisfy the SSP property permits to increase the stability range.

Acknowledgements Research partially supported by PRIN (2019-2021): "Innovative numerical methods for evolutionary partial differential equations and applications" and by INdAM-GNCS (2019) grant: "Approssimazione numerica di problemi di natura iperbolica ed applicazioni”.

Funding Open Access funding provided by Università degli Studi di Verona.

\section{Compliance with Ethical Standards}

Conflict of Interest On behalf of all authors, the corresponding author states that there is no conflict of interest. 
Open Access This article is licensed under a Creative Commons Attribution 4.0 International License, which permits use, sharing, adaptation, distribution and reproduction in any medium or format, as long as you give appropriate credit to the original author(s) and the source, provide a link to the Creative Commons licence, and indicate if changes were made. The images or other third party material in this article are included in the article's Creative Commons licence, unless indicated otherwise in a credit line to the material. If material is not included in the article's Creative Commons licence and your intended use is not permitted by statutory regulation or exceeds the permitted use, you will need to obtain permission directly from the copyright holder. To view a copy of this licence, visit http://creativecommons.org/licenses/by/4.0/.

\section{References}

1. Akrivis, G.: Implicit-explicit multistep methods for nonlinear parabolic equations. Math. Comput. 82, 45-68 (2012)

2. Akrivis, G., Crouzeix, M., Makridakis, C.: Implicit-explicit multistep methods for quasilinear parabolic equations. Numer. Math. 82, 521-541 (1999)

3. Albi, G., Dimarco, G., Pareschi, L.: Implicit-explicit multistep methods for hyperbolic systems with multiscale relaxation. arXiv:1904.03865 (2019)

4. Albi, G., Herty, M., Pareschi, L.: Linear multistep methods for optimal control problems and applications to hyperbolic relaxation systems. Appl. Math. Comput. 34, 460-477 (2019)

5. Ascher, U.M., Ruuth, S.J., Spiteri, R.J.: Implicit-explicit Runge-Kutta methods for time-dependent partial differential equations. Appl. Numer. Math. 25, 151-167 (1997)

6. Ascher, U.M., Ruuth, S.J., Wetton, B.T.R.: Implicit-explicit methods for time-dependent partial differential equations. SIAM J. Numer. Anal. 32, 797-823 (1995)

7. Bertozzi, A.L.: The mathematics of moving contact lines in thin liquid films. Not. AMS 45, 689-697 (1998)

8. Boscarino, S., Bürger, R., Mulet, P., Russo, G., Villada, L.M.: On linearly implicit IMEX Runge-Kutta methods for degenerate convection-diffusion problems modeling polydisperse sedimentation. Bull. Braz. Math. Soc. New Ser. 47(1), 171-185 (2016)

9. Boscarino, S., Filbet, F., Russo, G.: High order semi-implicit schemes for time dependent partial differential equations. J. Sci. Comput. 68, 975-1001 (2016)

10. Boscarino, S., Pareschi, L.: On the asymptotic properties of IMEX Runge-Kutta schemes for hyperbolic balance laws. J. Comput. Appl. Math. 316, 60-73 (2017)

11. Boscarino, S., Pareschi, L., Russo, G.: Implicit-explicit Runge-Kutta schemes for hyperbolic systems and kinetic equations in the diffusion limit. SIAM J. Sci. Comput. 35, A22-A51 (2011)

12. Boscarino, S., Pareschi, L., Russo, G.: A unified IMEX Runge-Kutta approach for hyperbolic systems with multiscale relaxation. SIAM J. Numer. Anal. 55(4), 2085-2109 (2017)

13. Carpenter, M.H., Kennedy, C.A.: Additive Runge-Kutta schemes for convection-diffusion-reaction equations. Appl. Numer. Math. 44(1/2), 139-181 (2003)

14. Dimarco, G., Pareschi, L.: Asymptotic-preserving IMEX Runge-Kutta methods for nonlinear kinetic equations. SIAM J. Numer. Anal. 51, 1064-1087 (2013)

15. Dimarco, G., Pareschi, L.: Implicit-explicit linear multistep methods for stiff kinetic equations. SIAM J. Numer. Anal. 55(2), 664-690 (2017)

16. Dimarco, G., Pareschi, L.: Numerical methods for kinetic equations. Acta Numerica 23, 369-520 (2014)

17. Filbet, F., Jin, S.: A class of asymptotic preserving schemes for kinetic equations and related problems with stiff sources. J. Comput. Phys. 229, 7625-7648 (2010)

18. Frank, J., Hundsdorfer, W., Verwer, J.G.: On the stability of implicit-explicit linear multistep methods. Appl. Numer. Math. 25, 193-205 (1997)

19. Gottlieb, S., Shu, C.-W., Tadmor, E.: Strong stability-preserving high-order time discretization methods. SIAM Rev. 43, 89-112 (2001)

20. Hundsdorfer, W., Ruuth, S.J.: IMEX extensions of linear multistep methods with general monotonicity and boundedness properties. J. Comput. Phys. 225, 2016-2042 (2007)

21. Hundsdorfer, W., Ruuth, S.J., Spiteri, R.J.: Monotonicity-preserving linear multistep methods. SIAM J. Numer. Anal. 41, 605-623 (2003)

22. Hundsdorfer, W., Verwer, J.: Numerical Solution of Time-Dependent Advection-Diffusion-Reaction Equations. Computational Mathematics. Springer, Berlin (2003)

23. Hairer, E., Wanner, G.: Solving Ordinary Differential Equation II: Stiff and Differential Algebraic Problems. Computational Mathematics, vol. 14. Springer, Berlin (1991). Second revised edition 1996 
24. Jin, S.: Efficient asymptotic-preserving (AP) schemes for some multiscale kinetic equations. SIAM J. Sci. Comput. 21(2), 441-454 (1999)

25. Naldi, G., Pareschi, L., Toscani, G.: Relaxation schemes for PDEs and applications to degenerate diffusion problems. Surv. Math. Ind. (Eur. J. Appl. Math.) 10, 315-343 (2002)

26. Pearson, J.E.: Complex patterns in a simple system. Science 261(5118), 189-192 (1993)

27. Pareschi, L., Russo, G.: Implicit-explicit Runge-Kutta schemes and applications to hyperbolic systems with relaxations. J. Sci. Comput. 25, 129-155 (2005)

28. Pareschi, L., Russo, G., Toscani, G.: A kinetic approximation to Hele-Shaw flow. Comptes Rendus Math. Acad. Sci. Ser. I 338(2), 178-182 (2004)

29. Pareschi, L., Zanella, M.: Structure preserving schemes for nonlinear Fokker-Planck equations and applications. J. Sci. Comput. 74(3), 1575-1600 (2018)

30. Rosales, R.R., Seibold, B., Shirokoff, D., Zhou, D.: Unconditional stability for multistep IMEX schemes: theory. SIAM J. Numer. Anal. 55(5), 2336-2360 (2017)

31. Seibold, B., Shirokoff, D., Zhou, D.: Unconditional stability for multistep IMEX schemes: practice. J. Comput. Phys. 376, 295-321 (2019)

32. Zhang, K., Wong, J.C.F., Zhang, R.: Second-order implicit-explicit scheme for the Gray-Scott model. J. Comput. Appl. Math. 213, 559-581 (2008) 